\title{
Reduced graphene oxide as metal-free catalyst for the light assisted Fenton-like reaction
}

\author{
Juan C. Espinosa, ${ }^{[b]}$ Sergio Navalón, ${ }^{[b]}$ Mercedes Álvaro, ${ }^{[b]}$ Hermenegildo García ${ }^{*[a]}$
}

\begin{abstract}
Natural Sunlight irradiation increases the catalytic activity of reduced graphene oxide ( $\mathrm{rGO}$ ) as metal-free Fenton-like catalyst for phenol degradation. Intermediacy of hydroxyl radicals was proved by electron paramagnetic resonance spectroscopy using a spin trapping agent.
\end{abstract}

\section{Introduction}

There is a considerable interest in exploiting the potential of graphenes (Gs) as metal-free catalysts. ${ }^{[1-5]}$ Gs in the absence of metals have been found to exhibit catalytic activity for a series of oxidations of benzylic compounds, ${ }^{[6,7]}$ alcohols $^{[6,8]}$ and amines ${ }^{[9]}$ as well as for hydrogenation of C-C multiple bonds ${ }^{[10]}$ or aromatic nitro compounds ${ }^{[11]}$ among other reactions. ${ }^{[2-4,12]}$ One of the challenges in carbocatalysis by Gs is to show that they can exhibit activity for reactions that up to now are believed to be promoted exclusively by metals such as C-C couplings, condensations, rearrangements and some types of oxidations. ${ }^{[2-}$ ${ }^{4]}$ In this regard the Fenton reaction consists in the generation of $\mathrm{HO}$ radicals in water by one electron reduction of $\mathrm{H}_{2} \mathrm{O}_{2}$. The typical reagent for the Fenton reaction is $\mathrm{Fe}^{2+}$ that is commonly used in stoichiometric amounts. ${ }^{[13,14]}$ We have recently reported that reduced graphene oxide $(\mathrm{rGO})$ is a suitable metal-free catalyst to promote the Fenton reaction at acid $\mathrm{pH}$ values $(\mathrm{pH}$ 3). ${ }^{[15]}$ Considering that $\mathrm{GO}$ and other types of Gs can be used as semiconductors ${ }^{[16]}$ and that an alternative to the Fenton reaction is the so-called photo-Fenton reaction, ${ }^{[14,17]}$ it is of interest to expand the previous report on the catalytic activity of rGO as Fenton catalyst to determine if light can also assist the Fenton reaction in the case of $\mathrm{rGO}{ }^{[15]}$ In the most simple photo-Fenton process, a Fe ${ }^{3+}$ salt in catalytic amounts is irradiated with UV-Vis light to promote the Fenton reaction. ${ }^{[14,18]}$ In the accepted reaction mechanism, irradiation of aqueous $\mathrm{Fe}^{3+}$ cation at wavelengths about $300 \mathrm{~nm}$ leads to the reduction of $\mathrm{Fe}^{3+}$ to $\mathrm{Fe}^{2+}$ and the generation of a hydroxyl radical due to the oxidation of a water molecule strongly solvating $\mathrm{Fe}^{3+}$ ion. ${ }^{[14,18]}$ Subsequently $\mathrm{Fe}^{2+}$ can react spontaneously with hydrogen peroxide forming $\mathrm{Fe}^{3+}$ and a second hydroxyl radical, closing the catalytic cycle.

[a] Prof. H.García

Departamento de Química and Instituto de Tecnología Química CSIC-UPV, Universidad Politécnica de Valencia, Consejo Superior de Investigaciones Científicas, Av. de los Naranjos s/n, 46022 Valencia, Spain; Center of Excellence for Advanced Materials Research, King Abdulaziz University, Jeddah, Saudi Arabia E-mail: hgarcia@qim.upv.es

[b] J.C.Espinosa, Dr.S.Navalón, Prof.M.Álvaro Departamento de Química and Instituto de Tecnología Química CSIC-UPV, Universidad Politécnica de Valencia, Consejo Superior de Investigaciones Científicas, Av. de los Naranjos s/n, 46022 Valencia, Spain

Supporting information for this article is given via a link at the end of the document.
Eqs. 1 and 2 show the two steps of the mechanism consisting in the photoinduced reduction of $\mathrm{Fe}^{3+}$ and spontaneous oxidation of $\mathrm{Fe}^{2+} \cdot[14,18,19]$

$$
\begin{gathered}
\mathrm{Fe}^{2+}+\mathrm{H}_{2} \mathrm{O}_{2} \underset{\mathrm{pH} 3\left(\mathrm{H}_{2} \mathrm{SO}_{4}\right)}{\longrightarrow} \mathrm{Fe}^{3+}+\mathrm{HO}^{-}+\mathrm{HO}^{-} \text {(Eq. 1) } \\
\mathrm{Fe}(\mathrm{OH})_{2}+\frac{\mathrm{UV}-\mathrm{Vis}}{\mathrm{pH} 3\left(\mathrm{H}_{2} \mathrm{SO}_{4}\right)} \mathrm{Fe}^{2+}+\mathrm{HO}
\end{gathered}
$$

Also in the case of $\mathrm{Au}^{[20-22]}$ and $\mathrm{Ag}^{[15]}$ nanoparticles (NPs) supported on diamond (D), it has been reported that natural Sunlight irradiation can assist the activity of these materials as Fenton catalysts. Au and Ag NPs exhibit a characteristic absorption band in the visible region generally known as surface plasmon band. ${ }^{[23]}$ Upon excitation of $\mathrm{Au}$ and Ag NPs at the surface plasmon bands several photophysical processes can take place, including electron photo-ejection from the metal NPs to $\mathrm{H}_{2} \mathrm{O}_{2}$ resulting in the generation of $\mathrm{HO} \cdot{ }^{[15,21]}$ In view of these precedents and also considering that $\mathrm{GO}$ and related $\mathrm{Gs}$ can also undergo photo-induced charge separation ${ }^{[24]}$ it is important to establish the influence of light in the catalytic activity of these materials as metal-free Fenton catalysts. In the present article we report that, also in the case of rGO, light assists the oneelectron reduction from $\mathrm{rGO}$ to $\mathrm{H}_{2} \mathrm{O}_{2}$.

\section{Results and Discussion}

In the initial stage of our work, phenol was selected as probe molecule to establish the occurrence of the Fenton reaction. Both phenol disappearance and $\mathrm{H}_{2} \mathrm{O}_{2}$ consumption were determined as a function of the reaction time. AFM measurements of the level of exfoliation of rGO under the reaction conditions show a distribution of sheets from 0.4 to 2.2 $\mathrm{nm}$ with an average of about $1 \mathrm{~nm}$ (see Fig. S1 in the supporting information). This corresponds to a situation in which the suspension contains from single to about four layers of rGO. Also in the preliminary state of the study, the possible influence of $\mathrm{Mn}$ on the light assisted Fenton-like phenol degradation was addressed. It is known that $\mathrm{GO}$ is contaminated by $\mathrm{Mn}^{2+}$ ions from the oxidation of graphite. We and others have determined that $\mathrm{Mn}$ content of $\mathrm{rGO}$ is in the range of $40 \mathrm{ppm}$. To address this issue, we performed a control experiment in which added on purpose Mn sulfate in amount corresponding to $2000 \mathrm{ppm}$. The results observed indicate no influence of the presence of $\mathrm{Mn}$ at these concentrations (see Fig. S2 in the supporting information). The influence of the initial $\mathrm{pH}$ value on the activity of rGO to promote the Fenton degradation of phenol in the dark or under natural sunlight irradiation is presented in Figs. 1 and S3. As it can be seen there, for $\mathrm{pH}$ values below than 5 rGO was able to promote phenol disappearance, increasing in activity as the initial $\mathrm{pH}$ value decreases in the range from 4.5 to 3.0. Importantly in all cases, both phenol disappearance and 
$\mathrm{H}_{2} \mathrm{O}_{2}$ consumption increases upon natural Sunlight irradiation with respect to a twin experiment in the dark (Fig. S3). Also blank controls in the absence of rGO by exposing phenol and $\mathrm{H}_{2} \mathrm{O}_{2}$ at $\mathrm{pH} 3$ to natural sunlight and in the presence of $\mathrm{rGO}$ exposed to natural sunlight but in the absence of $\mathrm{H}_{2} \mathrm{O}_{2}$ show negligible conversion as compared to the results observed in the presence of $\mathrm{rGO}$ (Fig. S4). These preliminary experiments shows convincingly that also for rGO Sunlight irradiation increases its catalytic activity for the Fenton-like reaction, shortening the time required for complete phenol degradation from $150 \mathrm{~h}$ in the dark to less than $7 \mathrm{~h}$ under sunlight illumination. This shorter time should be a consequence of the improved rate of $\mathrm{OH} \cdot$ generation from $\mathrm{H}_{2} \mathrm{O}_{2}$ similarly to the process shown in eq. 1. Moreover, irradiation with natural Sunlight can serve to mitigate somewhat the strong dependence of the Fenton catalytic activity with the $\mathrm{pH}^{[14,25]}$ In other words, while in the dark rGO only exhibits catalytic activity for $\mathrm{pH}$ values below 3.5, irradiation expands the $\mathrm{pH}$ range to 4.5 (Fig. 1 and S3), a fact that is important in wastewater treatment. Operation of sunlightassisted Fenton-like phenol degradation at somewhat higher $\mathrm{pH}$ values than the analogous dark process should be a consequence of the higher efficiency of $\mathrm{OH}^{-}$generation from $\mathrm{H}_{2} \mathrm{O}_{2}$ upon light irradiation.

In this context a continuous target in Fenton catalysis is to develop an efficient catalyst that can operate at $\mathrm{pH}$ values near neutrality with the minimum excess of $\mathrm{H}_{2} \mathrm{O}_{2}{ }^{[26-28]} \mathrm{H}_{2} \mathrm{O}_{2}$ is a relatively expensive commodity chemical and the amount used in a Fenton degradation process has to be optimized in order to ensure the economic attractiveness of the process. ${ }^{[26,}{ }^{27]}$ Accordingly we proceed to optimize the excess of $\mathrm{H}_{2} \mathrm{O}_{2}$ required for phenol degradation in the photo assisted Fenton reaction promoted by $\mathrm{rGO}$ at initial $\mathrm{pH}$ value of 3 . The results shown in Fig. 2 indicate that 5.5 equivalents of $\mathrm{H}_{2} \mathrm{O}_{2}$ are necessary to achieve complete phenol disappearance in less than $7 \mathrm{~h}$. Four equivalents of $\mathrm{H}_{2} \mathrm{O}_{2}$ result in a complete $\mathrm{H}_{2} \mathrm{O}_{2}$ consumption at 7 $\mathrm{h}$ without reaching complete phenol disappearance. Lower molar excesses of $\mathrm{H}_{2} \mathrm{O}_{2}$ lead to even lower phenol conversions at uncompleted $\mathrm{H}_{2} \mathrm{O}_{2}$ consumption.
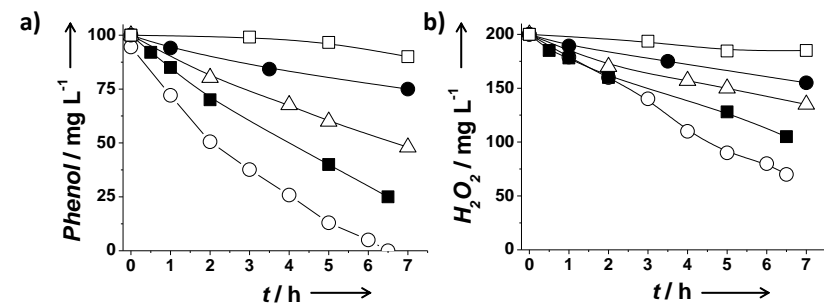

Figure 1. Phenol degradation (a) and $\mathrm{H}_{2} \mathrm{O}_{2}$ decomposition (b) using rGO as catalyst under natural Sunlight irradiation as a function of the initial $\mathrm{pH}$. Legend: $\mathrm{pH} 3(\circ), \mathrm{pH} 3.5(\bullet), \mathrm{pH} 4.0(\Delta), \mathrm{pH} 4.5(\bullet)$ and $\mathrm{pH} 5(\square)$. Reaction conditions: Catalyst $\left(200 \mathrm{mg} \mathrm{L}^{-1}\right)$, phenol (100 mg L-1; $\left.1.06 \mathrm{mM}\right), \mathrm{H}_{2} \mathrm{O}_{2}(200 \mathrm{mg}$ $\left.\mathrm{L}^{-1} ; 5.88 \mathrm{mM}\right), \mathrm{pH}$ as indicated in the legend.

It should be also commented that from the wastewater treatment point of view, besides phenol disappearance the corresponding

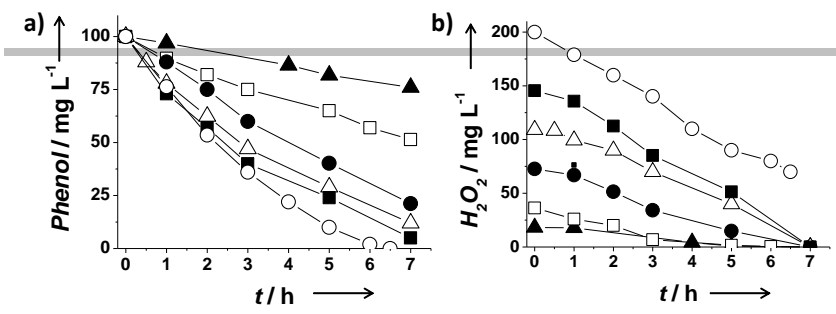

Figure 2. Influence of the $\mathrm{H}_{2} \mathrm{O}_{2}$ to phenol molar ratio for phenol degradation (a) and $\mathrm{H}_{2} \mathrm{O}_{2}$ decomposition (b) using $\mathrm{rGO}$ as catalyst under natural Sunlight irradiation. Legend of $\mathrm{H}_{2} \mathrm{O}_{2}$ to phenol molar ratio of $0.5(\Delta)$ ), $1(\square), 2(\bullet), 3(\Delta)$, 4 (a) and $5.5(\circ)$. Reaction conditions: Catalyst $\left(200 \mathrm{mg} \mathrm{L}^{-1}\right)$, phenol (100 mg $\left.\mathrm{L}^{-1} ; 1.06 \mathrm{mM}\right), \mathrm{H}_{2} \mathrm{O}_{2}$ to phenol molar ratio as indicated in the legend.

aromatic compounds formed as intermediates in the process should also be decomposed due to their even higher toxicity respect to phenol. ${ }^{[29]}$ In fact it is known that the attack of hydroxyl radicals on phenol results in the formation of hydroquinone, catechol and $p$-benzoquinone that are significantly more toxic than phenol. ${ }^{[15,29]}$ Therefore, the optimal $\mathrm{H}_{2} \mathrm{O}_{2}$ excess should not only lead to phenol disappearance but also to the decomposition of hydroquinone, catechol and $p$-benzoquinone. As it is shown in Figs. S5 and S6, while 5.5 equivalents of $\mathrm{H}_{2} \mathrm{O}_{2}$ result in complete phenol, hydroquinone, catechol and $p$-benzoquinone disappearance, the use of only 4 equivalents of $\mathrm{H}_{2} \mathrm{O}_{2}$ results in the presence of not only a residual concentration of phenol but, more importantly, in a significant concentration of hydroquinone accompanied by some catechol and p-benzoquinone (Fig. S6). Therefore, the minimum $\mathrm{H}_{2} \mathrm{O}_{2}$ excess for the process seems to be 5.5 equivalents. This low excess of $\mathrm{H}_{2} \mathrm{O}_{2}$ to degrade phenol and its aromatic reaction intermediates compares favorably with a related work that uses boron nitride as metal-free catalyst for the Fenton reaction that requires 11 equivalents. Importantly, rGO as metal-free (photo)catalyst behaves similarly than some of the most active photo-Fenton catalysts based on $\mathrm{Au}^{[21]}$ or $\mathrm{Ag}^{[30]} \mathrm{NPs}$ supported on functionalized diamond in terms of efficiency for $\mathrm{H}_{2} \mathrm{O}_{2}$ decomposition to hydroxyl radicals. Other works that report the use of metal-based catalyst have used $\mathrm{H}_{2} \mathrm{O}_{2}$ excess values around 1,000 or even $100,000 .{ }^{[27]}$

Overall, rGO decomposes efficiently $\mathrm{H}_{2} \mathrm{O}_{2}$ to $\mathrm{HO}$ radicals a process that has been further confirmed by EPR measurements and quenching experiments.

After having selected the $\mathrm{pH}$ value and the optimal $\mathrm{H}_{2} \mathrm{O}_{2}$ excess, a series of reuse experiments employing the same $\mathrm{rGO}$ sample in the presence of a large excess of phenol $(1,000 \mathrm{mg} \mathrm{L}$ $\left.{ }^{1}\right)$ respect to the catalyst employed $\left(200 \mathrm{mg} \mathrm{L}^{-1}\right)$ were also performed and the results are shown in Fig. 3 . As it can be seen there, rGO was able to promote the degradation of concentrated phenol aqueous solutions $\left(1,000 \mathrm{mg} \mathrm{L}^{-1}\right)$ in eight consecutive uses without observing a decrease in the catalytic activity. Comparison of the temporal profiles shown in Fig. 3 proves the photostability of rGO under photo-Fenton conditions. In fact, it was observed that the catalytic activity of used rGO increases gradually. Although reusability of some carbocatalysts under highly oxidative conditions of other advanced oxidation processes was found to be low, ${ }^{[2]}$ this is not the case under the present reaction conditions as evidenced in Fig. 3, probably due 
to the mild conditions of the experiment. Similar increase of catalytic activity was previously observed for rGO in the absence of light and this enhancement was attributed to slight modifications of rGO by introduction of hydroxyl groups on the rGO sheet. ${ }^{[15]} \mathrm{HO}$ radicals are among the most aggressive chemical species that can be present in water and they can virtually attack to any organic compound. ${ }^{[13,14]}$ Accordingly it is proposed that a certain proportion of $\mathrm{HO}$ radicals could attack rGO producing a certain degree of hydroxylation. ${ }^{[15]}$ The consequence of this hydroxylation is that upon reuse rGO becomes more hydrophilic and easily dispersible in water than the initial rGO, while still conveniently recoverable by filtration. In fact for the fourth use just simply magnetic stirring is sufficient to disperse completely used rGO catalyst making sonication unnecessary. This proposal is supported by chemical analysis comparing the $\mathrm{C} / \mathrm{O}$ atomic ratio of the fresh (5.9) and the five times used (2.7) rGO catalyst. Also IR spectroscopy shows for the five-times used rGO a clear increase in the intensity of the absorption band appearing about $1200 \mathrm{~cm}^{-1}$ corresponding to C$O$ stretching vibration, thus supporting an increase in the degree of rGO hydroxylation upon use (Fig. S7). This $1200 \mathrm{~cm}^{-1}$ band is accompanied by a very broad $\mathrm{O}-\mathrm{H}$ stretching absorption band from 3600 to $2700 \mathrm{~cm}^{-1}$ (see Fig. S7). C1s and O1s XPS of the fresh and five-times used rGO catalyst also shows the increase in the shift of the values to higher binding energies attributable to the introduction of oxygen functional groups in graphene (Fig. S8). Note that while the increase of O1s peak of rGO could suggest its partial conversion into $\mathrm{GO}$, the presence of $46.2 \%$ of graphenic $\mathrm{C}$ atoms in XPS peak shows that the sample should still be considered rGO. Thus, all the characterization techniques confirm that the morphology and main characteristics of rGO are maintained during exhaustive reuse, with some degree of additional hydroxylation. The fact that the rGO catalyst can be reused such a large number of runs in the presence of a large amount of phenol indicates the high activity and stability of rGO as photo-Fenton catalyst. However, as XPS is changing, it could be that, at even longer term, the photocatalytic activity of rGO changes, either deactivation or even reaching a stationary activity upon more extensive use. Apparently in those cases in which stability of carbocatalysts under advanced oxidation processes was found to be low, the flux of $\mathrm{OH}^{-}$radicals should be much higher than in the present conditions, causing deep damage to the carbon structure.

With regard to the reaction mechanism and particularly to provide evidence in support of the generation of $\mathrm{HO}$ radicals in the light assisted catalytic Fenton reaction promoted by rGO, electron paramagnetic resonance (EPR) studies using phenyl $\alpha$ tert-butyl nitrone (PBN) as spin trap were performed. PBN would react with generated $\mathrm{OH}^{\prime}$ giving a $\mathrm{PBN}-\mathrm{OH}$ radical that is stable and can be detected with steady-state EPR spectroscopy. The results are presented in Fig. 4. As it can be seen there, comparison of the experimental EPR spectrum with the simulated spectrum for the $\mathrm{PBN}-\mathrm{OH}$ adduct shows a good matching ${ }^{[31]}$ proving that $\mathrm{HO}$ radicals were generated in the light-assisted Fenton reaction by rGO.
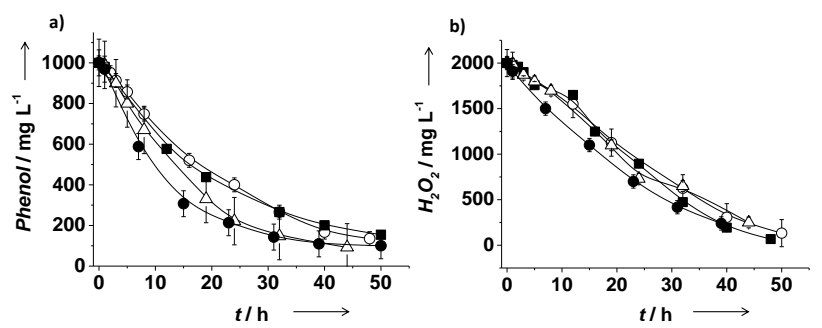

Figure 3. Productivity test for phenol degradation (a) and $\mathrm{H}_{2} \mathrm{O}_{2}$ decomposition (b) using rGO as catalyst under simulated Solar light irradiation. Legend: firs use $(\circ)$, second use $(\bullet)$, forth use $(\Delta)$ and eight use $(\bullet)$. Reaction conditions: catalyst $\left(200 \mathrm{mg} \mathrm{L}^{-1}\right)$, phenol $\left(1,000 \mathrm{mg} \mathrm{L}^{-1}\right), \mathrm{H}_{2} \mathrm{O}_{2}\left(2,000 \mathrm{mg} \mathrm{L}^{-1}\right), \mathrm{pH}_{3}$, simulated Sunlight irradiation.
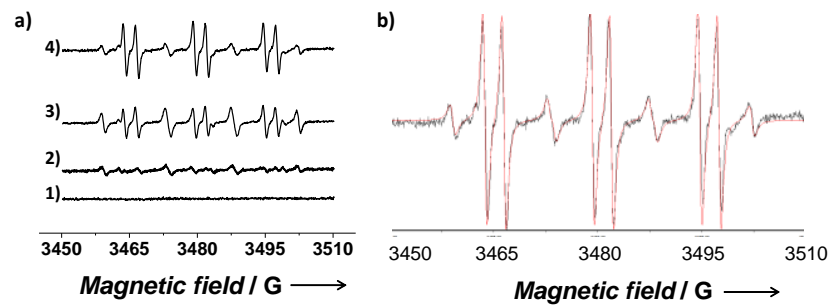

Figure 4. (a) EPR spectra of (1) $P B N+\mathrm{H}_{2} \mathrm{O}+$ Sunlight irradiation; (2) rGO + $\mathrm{PBN}+\mathrm{H}_{2} \mathrm{O}+$ Sunlight irradiation; (3) $\mathrm{rGO}+\mathrm{PBN}+\mathrm{H}_{2} \mathrm{O}_{2}+$ dark conditions; (4) $\mathrm{rGO}+\mathrm{PBN}+\mathrm{H}_{2} \mathrm{O}_{2}+$ Sunlight irradiation. (b) EPR spectra of sample (4) and its fitting considering the $\mathrm{PBN}-\mathrm{OH}$ adduct $(\mathrm{AGN}=15.5$ and $\mathrm{AGH}=2.7)$ and tertbutyl aminoxyl ( $\mathrm{AGN}=14.58$ and $\mathrm{AGH}=13.90$ ) coinciding with the reported values in the literature. ${ }^{[31]}$ EPR reaction conditions: $r G O\left(200 \mathrm{mg} \mathrm{L}^{-1}\right)$, PBN $\left(1,041 \mathrm{mg} \mathrm{L}^{-1}\right), \mathrm{PBN} / \mathrm{H}_{2} \mathrm{O}_{2}$ molar ratio $=1: 1, \mathrm{pH} 3,15$ min reaction time.

Another general way to support the involvement of $\mathrm{HO}$ radicals in a process is observation of the influence of dimethylsulfoxide (DMSO) as quencher. ${ }^{[20,31]}$ It is known in the literature that DMSO reacts with $\mathrm{HO}$ radicals with similar reaction rates as with phenol. ${ }^{[32]}$ Accordingly if phenol degradation by $\mathrm{H}_{2} \mathrm{O}_{2}$ is performed in the presence of increasing amounts of DMSO in excess with respect to phenol, then, phenol disappearance should proceed slower or eventually could stop depending on the excess of DMSO present as $\mathrm{HO}$ quencher in the medium. If DMSO is not added in excess amounts, then, due to the similar reaction rates of $\mathrm{OH}$. radicals with DMSO and phenol, a small effect of the presence of DMSO should make the quenching experiment not sufficiently conclusive. For the light-assisted Fenton reaction catalyzed by $\mathrm{rGO}$, an excess of DMSO results in an almost complete quenching of phenol degradation (Fig. S9).

When using rGO as metal free catalyst, one of the key issues is the nature of the active sites. ${ }^{[2,3,5]}$ In the original report describing the catalytic activity of $\mathrm{rGO}$ in the Fenton reaction, it was proposed that hydroquinone/p-benzoquinone-like moieties present in rGO due to the residual amount of oxygenated functional groups could act as reversible redox sites on rGO. ${ }^{[15]}$ Also in the present case of light assisted Fenton reaction, it is of interest to provide some data about the nature of active sites. Aimed at establishing a relationship between oxygen content and catalytic activity to additional samples with oxygen content between GO and rGO were prepared by 
partial reduction of GO with hydrazine ( 0.1 and $0.3 \%$ wt.) at room temperature. The results obtained (see Fig. S10 in the supporting information) clearly establish a general relationship between intermediate oxygen content and Fenton-like catalytic activity. The deeper the partial reduction of $\mathrm{GO}$ towards $\mathrm{rGO}$, the higher the catalytic activity. This is compatible with the formation of quinone/hydroquinone substructures by partial reconstruction of sp2 carbons from GO. Further support to the proposal of the nature of the active sites was also obtained by selecting hydroquinone and 2-methoxy hydroquinone as possible simple model molecules for the active sites present on rGO and studying their catalytic activity. An additional advantage of 2-methoxyhydroquinone is that it cannot derive from phenol oxidation and can be clearly be distinguished from phenol reaction products. On the other hand, while hydroquinone cannot be differentiated from phenol degradation product as the reaction progresses, the point is to compare the initial degradation rate in the absence of hydroquinone and in the presence of increasing initial amounts of this compound. As reported previously, the presence of a methoxy substituent decreasing the redox potential of the hydroquinone increases the catalytic activity in the dark. ${ }^{[15]}$ Importantly, natural Sunlight irradiation increases the catalytic activity observed for hydroquinone and 2-methoxyhydroquinone. Fig. S11 summarizes the catalytic activity of these two aromatic molecules for Fenton and photo-Fenton reactions. Quenching experiments with DMSO also confirm for these two simple aromatic molecules that $\mathrm{HO}$ radical are involved in the process (see Supporting Information). Therefore, the data presented in Fig. S11 supports that in the case of rGO those fragments having hydroquinone structure are upon light excitation active for Fenton phenol degradation.

Accordingly, a rationale mechanistic proposal parallel to those shown in equations 1 and 2 is proposed (Scheme 1) to rationalize the catalytic activity of $\mathrm{rGO}$ to promote Fenton degradation. Furthermore, some additional evidence in support of Scheme 1 was obtained by contacting independently hydroquinone or quinone with $\mathrm{H}_{2} \mathrm{O}_{2}$ and measuring the evolution of oxygen under the reaction conditions. While no oxygen evolution was measured for the reaction for hydroquinone and $\mathrm{H}_{2} \mathrm{O}_{2}$, the evolution of oxygen was observed for the reaction of quinone and $\mathrm{H}_{2} \mathrm{O}_{2}$ and the amount of $\mathrm{O}_{2}$ correlates with the initial concentration of quinone. The results are presented in Table S1 in the supporting information.

An alternative way to understand the activity of rGO as sunlight assisted Fenton like catalyst is based on the photocatalytic activity reported for $\mathrm{rGO}^{[33]}$ Therefore, upon light absorption, photogeneration of electron and holes would occur on rGO and, as indicated in Scheme 1, both electrons and holes will be quenched by $\mathrm{H}_{2} \mathrm{O}_{2}$, resulting in the generation of $\mathrm{OH}^{-}$in the semireaction in which electrons are transferred to $\mathrm{H}_{2} \mathrm{O}_{2}$. The $\mathrm{h}^{+}$ will be also quenched by $\mathrm{H}_{2} \mathrm{O}_{2}$ or $\mathrm{H}_{2} \mathrm{O}$ generating oxygen (see Scheme S1). However, the quenching of $h^{+}$by $\mathrm{H}_{2} \mathrm{O}_{2}$ seems more unlikely since hydrogen generation from water using rGO always require sacrificial electron donors to occur.
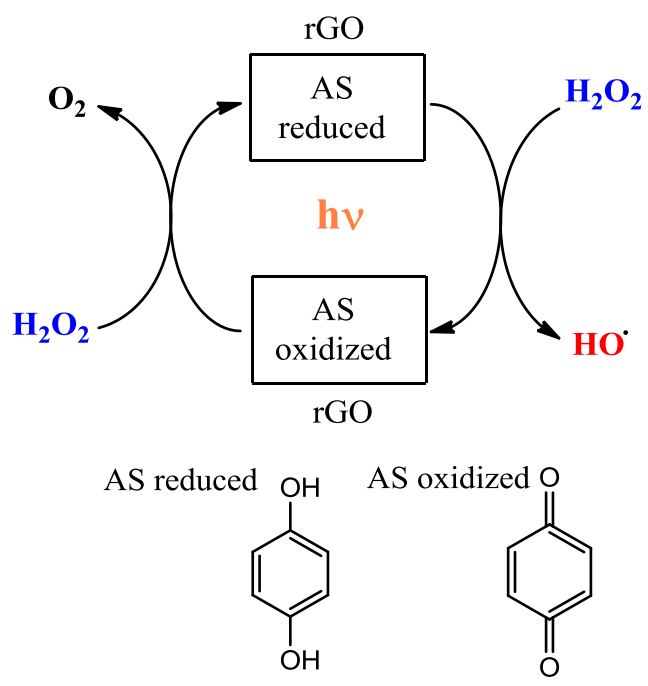

Scheme 1. Mechanistic proposal for the generation of hydroxyl radicals involving hydroquinone/p-benzoquinone as active sites (AS) in rGO.

Considering the accumulated total amount of phenol degraded in the eight runs of the productivity test ( $400 \mathrm{mg}$, see Fig. 3a) after subtracting the small relatively percentage $(5 \%$, see Fig. S4 in the supporting information) observed in blank runs in the absence of any rGO due to direct photochemical reaction of phenol and $\mathrm{H}_{2} \mathrm{O}_{2}$ and considering the oxygen content and its percentage that could correspond to quinone or hydroquinone based on XPS of the total rGO weight, an estimated turnover number of 383 is proposed. Of course this TON value is based on the assumptions about the nature of rGO active sites as associated to hydroquinone-quinone substructures and would include the dark (minor, see Fig S3) and light-assisted (major) processes promoted by rGO after discounting $5 \%$ of uncatalyzed degradation. It is also proposed that the dark and the light assisted phenol degradation taking place simultaneously in different proportion during the reaction occurs at similar oxygenated sites. Obviously, at the present level of understanding, this TON value has to be taken cautiously and, although it is still far from those reported for transition metals, clearly prove the catalytic nature of the process promoted by rGO.

Finally the catalytic activity of rGO for the light-assisted Fenton degradation of phenol was compared to that of other of related materials, namely GO, graphite and activated carbon (AC). The difference between $\mathrm{rGO}$ and $\mathrm{GO}$ is the oxygen content (about 46 and 12 wt\% for GO and rGO, respectively) and the structure of the sheet that in the case of rGO should have a considerable larger percentage of $\mathrm{sp} 2$ carbons with the existence of large regions of $\pi$ conjugation. ${ }^{[2,15,34]}$ As it can be seen in Fig. 5, catalytic activity of rGO is considerably larger than that of GO. Also in the case of GO an influence of light irradiation was observed as in the case of rGO, although in a considerably lesser degree. For graphite it was similarly observed a beneficial influence of Sunlight irradiation and the 

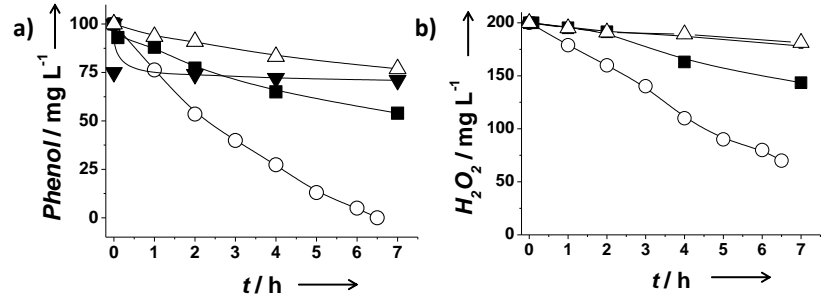

Figure 5. Phenol degradation (a) and $\mathrm{H}_{2} \mathrm{O}_{2}$ decomposition (b) using $\mathrm{rGO}(\mathrm{O})$, GO $(\Delta)$, graphite $(\boldsymbol{\square})$ or $A C(\boldsymbol{\nabla})$ as carbocatalysts. Reaction conditions: Catalyst $\left(200 \mathrm{mg} \mathrm{L}^{-1}\right)$, phenol $\left(100 \mathrm{mg} \mathrm{L}^{-1} ; 1.06 \mathrm{mM}\right), \mathrm{H}_{2} \mathrm{O}_{2}\left(200 \mathrm{mg} \mathrm{L}^{-1} ; 5.88\right.$ $\mathrm{mM}), \mathrm{pH} 3$, natural Sunlight irradiation.

resulting catalytic activity was intermediate between that of rGO and GO. Thus, although GO has shown under UV irradiation photocatalytic activity, for instance, for hydrogen generation, its low photoresponse under visible light irradiation due to its wider band gap limits the photocatalytic activity in the sunlight photoFenton degradation of phenol.

In the case of $A C$, no phenol degradation occurs in a significant extent as can be deduced from the time-conversion plot for $\mathrm{H}_{2} \mathrm{O}_{2}$ decomposition compared to phenol disappearance shown in Fig. 5. The initial decrease in phenol concentration taking place at very short contact times was due to physisorption of phenol on $\mathrm{AC}$ and part of this phenol could be recovered by extraction. The failure of $A C$ to act as carbocatalyst is compatible with the proposal of quinone/hydroquinone like substructures in graphene as redox pairs promoting Fenton-like phenol degradation. Note that in Fig. $5 b$ no $\mathrm{H}_{2} \mathrm{O}_{2}$ decomposition occurs on AC. Interestingly time profile shows for graphite an induction period. We attribute this induction period to the occurrence of a certain degree of spontaneous graphite exfoliation, by partial $\mathrm{H}_{2} \mathrm{O}_{2}$ oxidation leading to the evolution of some oxygenated graphene sheets in the aqueous phase, thus increasing the catalytic activity after a certain induction period. These $\mathrm{G}$ sheets together with $\mathrm{H}_{2} \mathrm{O}_{2}$ and light would form some hydroxylated $\mathrm{G}$ that structurally should be similar to $\mathrm{rGO}$, thus explaining its catalytic activity after an initial period.

\section{Conclusions}

Herein it has been shown that natural Sunlight also assists the catalytic activity of rGO for Fenton degradation of phenol with relatively low excess of $\mathrm{H}_{2} \mathrm{O}_{2}$. Activation with light expands about one $\mathrm{pH}$ unit towards neutral values the catalytic activity of rGO. Under these conditions, rGO even increases its efficiency upon reuse, a fact that has been attributed to the easier dispersability of used rGO. EPR spectroscopy and DMSO quenching support the generation of hydroxyl radicals and hydroquinone models suggest that this type of substructures could be responsible for the observed catalytic activity that would not be unique for rGO, but is a general property of other carbon based metal free catalyst whose activity depends on the density of active sites. Overall the present results constitute an additional example of the use of Gs as metal free catalyst, particularly in one reaction type that has been considered almost exclusive of transition metal catalysts. Future work is directed to expand the sunlight assisted Fenton like activity of rGO to other organic pollutants.

\section{Experimental Section}

Materials. Activated carbon (Norit SX Ultra, ref. 53663), graphite $(<20$ $\mathrm{mm}$, ref. 282863), $\mathrm{H}_{2} \mathrm{O}_{2}$ solution in water $(35 \%, \mathrm{v} / \mathrm{v})$, phenol $(>99 \%)$ $\mathrm{HNO}_{3}(65 \%), \mathrm{HCl}(37 \%)$ and $\mathrm{H}_{2} \mathrm{SO}_{4}(98 \%)$ were supplied by SigmaAldrich. The other reagents or solvents were of analytical or HPLC grade.

Catalyst preparation. The Gs employed in the present work include rGO and GO. These materials have been previously reported and fully characterized. ${ }^{[15]}$ In particular, GO was obtained by Hummers oxidation of graphite followed by exfoliation while rGO was produced by thermal reduction of $\mathrm{GO}$ at $200^{\circ} \mathrm{C}$.

Two samples with intermediate oxygen content between GO and rGO were obtained by past partial reduction of $\mathrm{GO}$ with different amounts of hydrazine. Briefly, an aqueous suspension $(50 \mathrm{~mL})$ of $\mathrm{GO}(1 \mathrm{mg} / \mathrm{mL})$ was stirred magnetically $(750 \mathrm{rpm})$ at $90^{\circ} \mathrm{C}$ and a given volume $(77 \mu \mathrm{L})$ of commercially available (Sigma Aldrich, 207942-500G) hydrazine $\left(\mathrm{H}_{2} \mathrm{~N}\right.$ $\mathrm{NH}_{2} \cdot \mathrm{H}_{2} \mathrm{O}, 64-65 \%$, reagent grade, $98 \%$ ) was added with a syringe and the mixture allowed to react two hours. After this time, the partially reduced GO samples were recovered from the suspension by centrifugation, washed exhaustively with water until neutral $\mathrm{pH}$, dried under vacuum at $50^{\circ} \mathrm{C}$, and used as catalyst under the general reaction conditions

Commercial AC and graphite were tested as-received as carbocatalysts for the photo-Fenton reaction.

Catalyst characterization. As commented fresh GO and rGO have been previously characterized. Herein we have characterized rGO after being tested for the photo-Fenton reaction using AFM, FT-IR, XPS and elemental analyses. AFM measurements to determine the level of exfoliation of rGO under reaction conditions were made by dropping 0.2 $\mu \mathrm{L}$ of the suspension on an atomically flat mica surface using a VEECO instrument in the Tapping Mode. In particular, a SPECS spectrometer with a Phoibos 150 MCD-9 detector using a non-monochromatic X-ray source ( $\mathrm{Al}$ and $\mathrm{Mg}$ ) operating at $200 \mathrm{~W}$ was employed to record the XPS spectra. Prior to the analysis the samples were evacuated at $1 \cdot 10^{-9} \mathrm{mbar}$ in the pre-chamber of the spectrometer. CASA software was employed to perform the spectra deconvolution and component quantification. ATRFTIR spectra were recorded by using a Bruker Tensor27 instrument. rGO the sample was pre-treated in an oven at $100{ }^{\circ} \mathrm{C}$ for $12 \mathrm{~h}$ to remove physisorbed water. Prior to recording the spectra the samples were equilibrated at room temperature. Elemental analyses were measured using a Perkin-Elmer CHNOS analyzer.

Photocatalytic experiments. The photocatalytic experiments were carried out at least in triplicate (June-July, 2015, Technical University of Valencia, Valencia, Spain). To a round-bottom flask $(500 \mathrm{~mL})$ containing an aqueous phenol solution ( $25 \mathrm{~mL} ; 100 \mathrm{mg} \mathrm{L}^{-1}$ ) the required amount of the solid catalyst $\left(200 \mathrm{mg} \mathrm{L}^{-1}\right)$ was added and the system sonicated for 2 $\mathrm{h}$. Then, the initial $\mathrm{pH}$ value of the aqueous suspension was adjusted to the required value using $\mathrm{HNO}_{3}(0.1 \mathrm{M})$ or $\mathrm{NaOH}(0.1 \mathrm{M})$. At this time the samples were exposed to the natural Sunlight irradiation for at least 30 min to equilibrate the temperature. The reactions were carried out at the open air. The reaction starts once the required amount of $\mathrm{H}_{2} \mathrm{O}_{2}$ was 
added. During the course of the reaction different aliquots were sampled and filtered through a $0.2 \mu \mathrm{m}$ Nylon to remove the carbonaceous catalyst. The natural Sunlight radiation intensity was measured using a photodiode connected to a voltmeter. In average the solar irradiation and the temperature were $920 \pm 14 \mathrm{~mW} \mathrm{~cm}^{-2}$ and $29 \pm .2 .2 \stackrel{\circ}{\circ}$, respectively.

In the case of homogeneous photo-Fenton experiments using hydroquinone of 2-methoxyhydroquinone a concentration of $0.106 \mathrm{mM}$ was employed as organocatalyst instead the use of the solid carbonaceous material.

Productivity test were performed using a large amount of phenol $(1,000$ $\left.\mathrm{mg} \mathrm{L}^{-1}\right)$ and $\mathrm{H}_{2} \mathrm{O}_{2}\left(2,000 \mathrm{mg} \mathrm{L}^{-1}\right)$ respect to the catalyst $\left(200 \mathrm{mg} \mathrm{L}^{-1}\right)$ at $\mathrm{pH}$ 3. To study the reusability of the carbocatalyst, rGO was filtered through a membrane filter and washed with a basic aqueous solution $(\mathrm{pH}$ 10 ) and then with Milli- $Q$ water to remove possible adsorbed organic compounds. Then, a new photocatalytic reaction was performed. To avoid possible daily variations we performed the photocatalytic experiments using simulated Sunlight irradiation (Oriel® Sol1A). The temperature of the reaction was around $22{ }^{\circ} \mathrm{C}$ with an irradiation intensity of 1 Sun.

Analysis of phenol, catechol, hydroquinone and $p$-benzoquinone. The filtered reaction aliquots were analysed by HPLC using a reverse phase stationary column (Kromasil-C18) working under isocratic conditions $\left(69 / 30 / 1 \mathrm{v} \%, \mathrm{H}_{2} \mathrm{O} / \mathrm{CH}_{3} \mathrm{CN} / \mathrm{CH}_{3} \mathrm{COOH}\right)$ and using a photodiode array detector. The samples of the productivity test were 10 -fold diluted in Milli-Q water before analysis.

$\mathrm{H}_{2} \mathrm{O}_{2}$ measurements. The previously filtered sample aliquots were 20fold diluted, or 200-fold diluted in the productivity test samples, and $\mathrm{K}_{2}(\mathrm{TiO})\left(\mathrm{C}_{2} \mathrm{O}_{4}\right)_{2}$ in $\mathrm{H}_{2} \mathrm{SO}_{4} / \mathrm{HNO}_{3}$ was added as colorimetric titrating agent and allowing to react for $10 \mathrm{~min}$. The intensity of the developed yellow colour was monitored at $420 \mathrm{~nm}$.

Oxygen evolution measurement. To an aqueous solution of quinone (22 and $44 \mathrm{mg} / \mathrm{L}$ ) or hydroquinone (22 mg/L), $\mathrm{H}_{2} \mathrm{O}_{2}$ (400 and $800 \mathrm{mg} / \mathrm{L}$ ) was added in a single step and the reaction was performed in a sealed round bottom flask connected to an inverted burette to collect gases. The reaction was followed for 5 hours at room temperature upon sun simulated light. No gas evolution was observed in the case of hydroquinone, while oxygen evolution was observed for quinone, and the volume was related with the concentration of the cyclic diketone

Selective hydroxyl radical quenching experiments and EPR measurements. DMSO was added to the photocatalytic system as selective hydroxyl radical scavenger under the following conditions: 200 $\mathrm{mg} \mathrm{L}^{-1} \mathrm{rGO}, 100 \mathrm{mg} \mathrm{L}^{-1}$ phenol, $200 \mathrm{mg} \mathrm{L}^{-1} \mathrm{H}_{2} \mathrm{O}_{2}$, DMSO to $\mathrm{H}_{2} \mathrm{O}_{2}$ molar ratio $10, \mathrm{pH} 3$ and natural Sunlight irradiation.

EPR measurements were carried out using PBN as spin trap under the following reaction conditions: catalyst $\left(200 \mathrm{mg} \mathrm{L}^{-1}\right)$, PBN $\left(1,000 \mathrm{mg} \mathrm{L}^{-1}\right)$, $\mathrm{H}_{2} \mathrm{O}_{2}$ to $\mathrm{PBN}$ molar ratio $1, \mathrm{pH} 3$, dark or natural Sunlight irradiation, reaction time $(15 \mathrm{~min})$. Filtered $(0.2 \mu \mathrm{m})$ and nitrogen-purged aliquots were measured using a Bruker EMX using the following typical settings: frequency $9.803 \mathrm{GHz}$, sweep width $3489.9 \mathrm{G}$, time constant $40.95 \mathrm{~ms}$, modulation frequency $100 \mathrm{kHz}$, modulation width $1 \mathrm{G}$, microwave power $19.92 \mathrm{~mW}$.

\section{Acknowledgements}

Financial support by the Spanish Ministry of Economy and Competitiveness (Severo Ochoa and CTQ2014-53292-R) is gratefully acknowledged. Generalidad Valenciana is also thanked for funding (Prometeo 2013/014).

Keywords: Photocatalysis - heterogeneous catalysis $\cdot$ reduced graphene oxide $\bullet$ Fenton- reaction $\bullet$ natural Sunlight

[1] D. R. Dreyer, R. S. Ruoff, C. W. Bielawski, Angew. Chem. Int. Ed. 2010, 49, 9336 - 9344; D. R. Dreyer, C. W. Bielawski, Chem. Sci. 2011, 2, 1233-1240; X.-K. Kong, C.-L. Chen, Q.-W. Chen, Chem. Soc. Rev. 2014, 43, 2841-2857; Y. Zhai, Z. Zhu, S. Dong, ChemCatChem 2015 , 7, 2806-2815; H. Hu, J. H. Xin, H. Hu, X. Wang, Y. Kong, Appl. Catal. B 2015, 492, 1-9.

[2] S. Navalon, A. Dhakshinamoorthy, M. Alvaro, H. Garcia, Chem. Rev. 2014, 114, 6179-6212.

[3] C. Su, K. P. Loh, Acc. Chem. Res. 2013, 46, 2275-2285.

[4] D. S. Su, S. Perathoner, G. Centi, Chem. Rev. 2013, 113, 5782-5816.

[5] J. Albero, H. Garcia, J. Mol. Catal. A-Chem. 2015, 408, 296-309.

[6] D. R. Dreyer, H.-P. Jia, C. W. Bielawski, Angew. Chem. Int. Ed. 2010, 49, $6813-6816$

[7] A. Dhakshinamoorthy, A. Primo, P. Concepción, M. Alvaro, H. García, Chem. Eur. J. 2013, 19, 7547-7554; Y. Gao, G. Hu, J. Zhong, Z. Shi, Y. Zhu, D. S. Sheng Su, J. Wang, X. Bao, D. Ma, Angew. Chem. Int. Ed. 2013, 52, 2109 -2113; G. Lv, H. Wang, Y. Yang, T. Deng, C. Chen, Y. Zhu, X. Hou, ACS Catal. 2015, 5, 5636-5646.

[8] J. Long, X. Xie, J. Xu, Q. Gu, L. Chen, X. Wang, ACS Catal. 2012, 2, 622-631.

[9] C. Su, M. Acik, K. Takai, J. Lu, S.-j. Hao, Y. Zheng, P. Wu, Q. Bao, T. Enoki, Y. J. Chabal, K. P. Loh, Nat. Comm. 2012, Art. n ${ }^{\circ} 1298 ;$ X.-H. Li, M. Antonietti, Angew. Chem. Int. Ed. 2013, 52, 4572-4576.

[10]T. I. Perhun, Bychko, I.B., Trypolsky, A.I., Strizhak, P.E., Theor. Exp. Chem. 2013, 48, 367-370; A. Primo, F. Neatu, M. Florea, V. Parvulescu, H. Garcia, Nat. Commun. 2014, 5, art. no 5291.

[11] Y. Gao, D. Ma, C. Wang, J. Guan, X. Bao, Chem. Commun. 2011, 47, 2432-2434.

[12]B. Basu, S. Kundu, D. Sengupta, RSC Adv. 2013, 3, 22130-22134; V. Schwartz, W. Fu, Y.-T. Tsai, H. M. Meyer III, A. J. Rondinone, J. Chen, Z. Wu, S. H. Overbury, C. Liang, ChemSusChem 2013, 6, 840846; J.-H. Yang, Sun, G., Gao, Y., Zhao, H., Tang, P., Tan, J., Lu, A.H., Ma, D., Energy Environ. Sci. 2013, 6, 793-798.

[13]E. Neyens, Baeyens, J., J. Hazard. Mater. 2003, 98, 33-50.

[14] J. J. Pignatello, E. Oliveros, A. Mackay, Crit. Rev. Environ. Sci. Technol. 2006, 36, 1-84.

[15] J. C. Espinosa, S. Navalón, A. Primo, M. Moral, J. Fernández Sanz, M Álvaro, H. García, Chem. Eur. J. 2015, 21, 11966-11971.

[16]Z. Sun, Q. Ye, C. Chi, J. Wu, Chem. Soc. Rev. 2012, 41, 7857-7889; Q Xiang, J. Yu, M. Jaroniec, Chem. Soc. Rev. 2012, 41, 782-796; N. Zhang, M.-Q. Yang, S. Liu, Y. Sun, Y.-J. Xu, Chem. Rev. 2015, 115 , 10307-10377; M.-Q. Yang, N. Zhang, M. Pagliaro, Y.-J. Xu, Chem.Soc.Rev. 2014, 43, 8240-8254; N. Zhang, X. Y.-J., CrystEngComm 2016, 18, 24-37.

[17] K. Ayoub, E. D. van Hullebusch, M. Cassir, A. Bermond, J. Hazard. Mater. 2010, 178, 10-28; M. N. Chong, B. Jin, C. W. K. Chow, C. Saint, Water Res. 2010, 44 2997-3027; M. Pera-Titus, V. GarcíaMolina, M. A. Baños, J. Giménez, S. Esplugas, Appl. Catal. B 2004, 47, 219-256; S. Rahim Pouran, Abdul Aziz, A.R., Wan Daud, W.M.A., J. Ind. Eng. Chem. 2015, 21, 53-69.

[18]I. P. Pozdnyakov, E. M. Glebov, V. F. Plyusnin, W. P. Grivin, Y. V. Ivanov, D. Y. Vorobyev, N. Bazhin, Pure Appl. Chem. 2000, 72, $2187-$ 2197.

[19] M. Pera-Titus, V. García-Molina, M. A. Baños, J. Giménez, S. Esplugas, Appl. Catal. B 2004, 47, 219-256. 
[20] S. Navalon, Martin, R., Alvaro, M., Garcia, H., ChemSusChem 2011, 4 , 650-657.

[21]S. Navalon, M. De Miguel, R. Martin, M. Alvaro, H. Garcia, J. Am. Chem. Soc. 2011, 133 2218-2226.

[22]D. Sempere, S. Navalon, M. Dančíková, M. Alvaro, H. Garcia, Appl. Catal. B 2013, 142-143, 259-267.

[23] J. C. Scaiano, J. C. Netto-Ferreira, E. Alarcon, P. Billone, C. J. BuenoAlejo, C. O. L. Crites, M. R. Decan, C. Fasciani, M. Gonzalez-Bejar, G. L. Hallett-Tapley, M. Grenier, K. L. McGilvray, N. L. Pacioni, A Pardoe, L. Rene-Boisneuf, R. Narbonne-Schwartz, J. Silvero, K. G. Stamplecoskie, T. L. Wee, Pure Appl. Chem. 2011, 83, 913-930; J. C. Scaiano, K. G. Stamplecoskie, G. L. Hallett-Tapley, Chem. Commun. 2012, 48, 4798-4808.

[24]F. D'Souza, O. Ito, Sci. Prog. 2013, 96, 369-397; J. D. Roy-Mayhew, I A. Aksay, Chem. Rev. 2014, 114, 6323-6348.

[25]A. D. Bokare, W. Choi, J. Hazard. Mater. 2014, 275, 121-135.

[26] A. Dhakshinamoorthy, S. Navalon, M. Alvaro, H. Garcia, ChemSusChem 2012, 5, 46-64; S. Navalon, M. Alvaro, H. Garcia, Appl. Catal. B 2010, 99, 1-26.

[27] S. Navalon, A. Dhakshinamoorthy, M. Alvaro, H. Garcia, ChemSusChem 2011, 4, 1712-1730.
[28]S. Navalon, A. Dhakshinamoorthy, M. Alvaro, H. Garcia, Coord. Chem. Rev. (http://dx.doi.org/10.1016/j.ccr.2015.12.005 2015.

[29] J. A. Zazo, J. A. Casas, C. B. Molina, A. Quintanilla, J. J. Rodriguez, Environ. Sci. Technol. 2007, 41 7164-7170.

[30] J. C. Espinosa, S. Navalón, M. Moral, M. Álvaro, H. García, ChemCatchem 2015, 7, 2682-2688.

[31] M. J. Burkitt, R. P. Mason, Proc. Natl. Acad. Sci. USA 1991, 88, 8440 8444.

[32]Y.-N. Wang, J. Chen, X. Li, S. Zhang, X. Qiao, QSAR Comb. Sci. 2009, 28, 1309-1316; J. S. Beckman, T. W. Beckman, J. Chen, P. A. Marshall, B. A. Freeman, Proc. Natl. Acad. Sci. USA 1990, 87, 16201624.

[33]C. Chen, W. Cai, M. Long, B. Zhou, Y. Wu, D. Wu, Y. Feng, ACS Nano 2010, 4, 6425-6432; T. F. Yeh, C. Y. Teng, S. J. Chen, H. Teng, Adv. Mater. 2014, 26, 3297-3303; T.-F. Yeh, F.-F. Chan, C.-T. Hsieh, H. Teng, J. Phys. Chem. C 2011, 115, 22587-22597.

[34]D. R. Dreyer, S. Park, C. W. Bielawski, R. S. Ruoff, Chem. Soc. Rev. 2010, 39, 228-240. 


\section{Entry for the Table of Contents}

\section{FULL PAPER}

Reduced graphene oxide acts as photo Fenton catalyst generating hydroxyl radicals from $\mathrm{H}_{2} \mathrm{O}_{2}$ upon sunlight irradiation

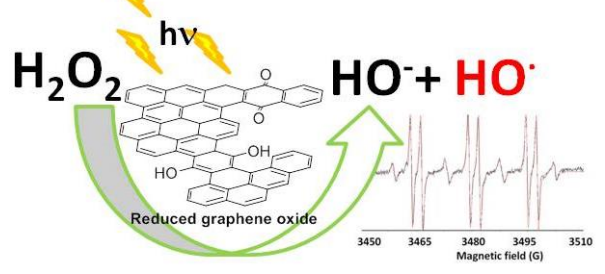

Juan C. Espinosa, Sergio Navalón, Mercedes Álvaro, Hermenegildo García*

Page No. - Page No.

Reduced graphene oxide as metalfree catalyst for the light assisted Fenton reaction 